\title{
Disturbances of C-fibre-mediated sensibility in lumbosacral disc disease
}

\author{
F Strian, S Lautenbacher, G Karlbauer, G Galfe
}

\begin{abstract}
In nine patients with chronic lumbosacral disc disease and radicular symptoms clearly restricted to one leg, C-fibre-mediated sensibility was measured by determination of the thresholds for heat pain and warmth on the foot, ipsi- and contralaterally to the nerve root compression. The thresholds were compared with the values for 19 healthy subjects. In the patients the warmth threshold was increased in the ipsilateral dermatome and normal in the contralateral dermatome. In contrast, the heat pain threshold was near normal ipsilaterally but was clearly decreased contralaterally. These findings are discussed with respect to a possible pain sensitisation resulting from nerve root compression.
\end{abstract}

Disturbances of sensibility are one of the main symptoms in lumbosacral disc disease. ${ }^{1}$ Nevertheless, experimental attempts to quantify the type and intensity of these sensory deficits have been rare. In patients with lumbosacral disc herniation distinct right-left differences were found in the thresholds for pressure and vibration on the lower extremities, a finding that could not be obtained with the usual pinprick and light touch testing. ${ }^{2}$ Moreover, with electrocutaneous stimulation sensory threshold increases were observed ipsilaterally to the lumbosacral nerve root compression. ${ }^{3} \mathrm{~A}$ study with radiation heating ${ }^{4}$ suggested that C-fibre-mediated sensibility is also affected, increased pain thresholds being found in the affected dermatomes of patients with acute radiculopathy.

The aim of this pilot study was to quantify the disturbances of C-fibre-mediated cutaneous sensibility (heat pain, warmth) in patients with chronic lumbosacral disc disease whose radicular symptoms were clearly confined to one leg. The sensory thresholds were measured ipsi- and contralaterally to the putative root compression and compared with the thresholds of healthy subjects.

Method

From a larger group of patients with chronic disc disease (duration of symptoms at least one year) we selected nine patients (five women and four men) between the ages of 23 and 44 years (mean age 36.9) who had lumbosacral radiculopathy (L5 or S1) and whose motor and sensory symptoms were clearly restricted to one leg. Definite asymmetrical sensory impairment (diminished sensitivity to pinprick, light touch, and vibration), motor deficits (pareses and reduced reflexes related to the affected nerve root) and Lasegue's sign were present on the affected side. The sensory deficits were documented in all nine patients by function tests of the large myelinated fibres (vibration or light touch) and in seven patients by the test of the small myelinated fibres (pinprick). In all patients the test was performed on both legs, but deficits were found only in the symptomatic leg. The reports on pain radiation into the leg corresponded with the location of the sensory deficits. The mean duration of back pain, which all patients described as recurrent pain attacks, was seven years (range: three to 20 years); seven patients rated the intensity of their most recent pain attack as very severe, one as fairly severe, and one as moderate. The diagnoses, based on computerised tomography, were disc prolapse in five cases, disc protrusion in two, spinal stenosis in one, and no major findings in one. Additionally, 19 healthy control subjects (nine women and 10 men) between the ages of 22 and 63 years (mean $=44.3$ ) were invesigated.

Heat pain and warmth thresholds were measured on one site on each foot: on the dermatome of the putative nerve root compression and on the contralateral dermatome of the same segment. The stimulator used was a temperature controlled Peltier thermode with an active surface of $6 \mathrm{~cm}^{2}$ (modified Marstock thermode 5 . For measuring heat pain eight stimuli were applied as follows: starting from $40^{\circ} \mathrm{C}$ a temperature rise of $0.8^{\circ} \mathrm{C} / \mathrm{s}$ was started automatically and continued until the subject felt pain for the first time and pressed the response button, the base temperature was then re-established. The mean of the last five trials was taken as the threshold measure. For determination of the warmth threshold seven stimuli were administered in the same manner, but starting from $32^{\circ} \mathrm{C}$. The subject was told to press the response button as soon as he or she detected an increase in temperature. 
Table Mean (SD) threshold values $\left({ }^{\circ} \mathrm{C}\right)$ for nine patients measured in dermatome of putative root compression (IPSI) and in contralateral dermatome of same segment (CONTRA). Values for 19 controls also shown

\begin{tabular}{llcc}
\hline & \multicolumn{2}{l}{ Patients } & \\
\cline { 2 - 3 } Threshold & IPSI & CONTRA & \multicolumn{1}{c}{ Controls } \\
\hline Warmth & $11 \cdot 1(3 \cdot 4)^{\star}$ & $7 \cdot 2(3 \cdot 1)$ & $7 \cdot 6(3.0)$ \\
Heat pain & $48 \cdot 1(1 \cdot 6)^{\star \star}$ & $45 \cdot 8(1 \cdot 2)^{\star \star \star}$ & $47 \cdot 3(1 \cdot 8)$
\end{tabular}

*IPSI > CONTRA, $p=0.011$ (Wilcoxon signed-rank test); IPSI > NORM, $p=0.015$ (Mann-Whitney $U$ test). $\star \star$ IPSI $>$ CONTRA, $p=0.008$ (Wilcoxon signed-rank test) $\star \star \star$ CONTRA $<$ NORM, $p=0.032$ (Mann-Whitney $U$ test)

The mean of the seven trials was used as the threshold measure. With this method no right-left differences or differences between the dermatomes of the segments L5 and S1 were found in the healthy control subjects.

\section{Results}

The threshold values of the patients and the control subjects and the results of the statistical testing are shown in the table. The warmth thresholds of the patients were significantly increased on the dermatome of the nerve root compression compared with the thresholds of the contralateral dermatome of the same segment. The contralaterally observed thresholds did not differ from those of the control subjects. The results for the heat pain thresholds were different. In the patients the difference between the pain thresholds measured ipsilaterally and contralaterally to the root compression was significant and had the same sign as that for the warmth threshold. Whereas the ipsilateral values were only slightly greater than those of the control subjects (NS), however, the contralateral values were significantly smaller.

\section{Discussion}

Our findings suggest that for C-fibremediated cutaneous sensibility the sensory modalities heat pain and warmth are affected differently by a long-term lumbosacral nerve root compression. The sensibility for warmth was disturbed in a way that might be expected if the root compression is thought of as a long term nerve blockade: the thresholds were increased in the dermatome of the affected root but normal in the contralateral dermatome of the same segment. In contrast, the threshold increase in heat pain perception on the dermatome belonging to the affected root was quite small; the greater alteration was a contralateral threshold decrease, which is suggestive of some kind of sensitisation.
One interpretation is that all primary afferent fibres (large myelinated, small myelinated, and unmyelinated) are affected by a root compression and the involvement of large fibres leads to a weakening of the spinal pain inhibiting system. Such a system has been postulated as part of the gate control theory, and its existence has been established empirically. ${ }^{6}$ Animal studies suggest that the pain inhibiting effect on dorsal horn neurons of a stimulation of large fibres is mediated by opioidergic interneurons via pre- and postsynaptic mechanisms. ${ }^{7}$ On the side with the root compression the combination of decreased inhibitory control and nociceptive activity might have resulted in the neglible increase in pain threshold, whereas contralaterally, with normal nociceptive activity, the loss of the inhibitory counterbalance might have led to a clearly decreased pain threshold-that is, pain sensitisation. In support of this assumption, in studies with transcutaneous nerve stimulation the opposite effect was found: the pain sensitivity in the limb contralaterally to the stimulation was reduced. ${ }^{89}$

We have already postulated such a mechanism of localised pain sensitisation, which has effects in one or more adjacent spinal segments, and its involvement in the pathogenesis of chronic pain. ${ }^{10}$ Our results indicate that additional systematic research based on this hypothesis would be promising.

We thank the Fachklinik Enzensberg, and Drs A Weichenmeier and $W$ Schupp for recruiting the subjects with disc disease, and for providing organisational assistance in connection with the study.

1 Mumenthaler M, Schliack H. Läsionen peripherer Nerven: Diagnostik und Therapie. Stuttgart: Georg Thieme Verlag, 1987.

2 Weise MD, Garfin SR, Gelberman RH, Katz MM, Thorne RP. Lower-extremity sensibility testing in patients with herniated lumbar intervertebral discs. J Bone Joint Surg herniated lumbar

3 Katif HA, Sedgwick EM. Evaluation of the dermatomal somatosensory evoked potential in the diagnosis of lumbosacral root compression. J Neurol Neurosurg Psychiatry 1987;50:1204-10.

4 Blumenkopf $\mathrm{B}$, Lipman JJ. Objective assessment of the nociceptive sensibility and deficits associated with radiculopathy using the heat beam dolorimeter. Pain 1987;suppl 4:10.

5 Fruhstorfer H, Lindblom U, Schmidt G. Method for quantitative estimation of thermal thresholds in patients. J Neurol Neurosurg Psychiatry 1976;39:1071-5.

6 Price DD. Psychological and neural mechanisms of pain. New York: Raven Press, 1988.

7 Yaksh TL. Spinal afferent processing. New York: Plenum Press, 1986.

8 Callaghan M, Sternbach RA, Nyquist JK, Timmermans G. Changes in somatic sensitivity during transcutaneous Changes in somatic sensitivity during
electrical analgesia. Pain 1978;5:115-27.

9 Malow RM, Dougher MJ. A signal detection analysis of the effects of transcutaneous stimulation on pain Psychosomatic Med 1979;41:101-8.

10 Strian F. Diagnostische und klinische Aspekte der Neuropathie der kleinen Nervenfasern. Fortschritte der Neurologie und Psychiatrie 1990;58:51-65. 